\title{
Molecular organization relationship of low-bandgap polymers at the air- water interface and in solid films
}

\author{
Vinicius Jessé Rodrigues de Oliveira ${ }^{\mathrm{a}, *}$, Edilene Assunção da Silva ${ }^{\mathrm{a}, \mathrm{c}}$, Maria Luisa Braunger ${ }^{\mathrm{b}}$, Hussein Awada ${ }^{\mathrm{c}}$, \\ Henrique de Santana ${ }^{d}$, Roger C. Hiorns ${ }^{c}$, Christine Lartigau-Dagron ${ }^{\text {c }}$, Clarissa de Almeida Olivati ${ }^{\mathrm{a}, *}$ \\ a Laboratório de Optoeletrônica e Filmes Finos, Departamento de Física, Faculdade de Ciências e Tecnologia, UNESP, Rua Roberto Simonsen, 305, Presidente Prudente, SP 19060-900, Brazil

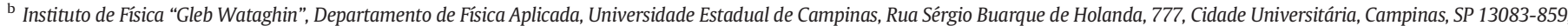 \\ Brazil \\ c CNRS/Univ Pau E' Pays 'Adour, Institut des Sciences Analytiques et Physico-Chimie pour l'Environnement et les Materiaux, Pau, France \\ ' Centro de Ciências Exatas, Departamento de Química, Universidade Estadual de Londrina, Londrina, PR 86057-970, Brazil
}

\section{A R T I C L E I N F O}

\section{Article history:}

Received 19 March 2018

Received in revised form 13 June 2018

Accepted 4 July 2018

Available online 6 July 2018

\section{Keywords:}

Low-bandgap polymers

Langmuir films

Solid thin films

Energy diagram

Photoconductivity effect

\begin{abstract}
A B S T R A C T
Low-bandgap organic polymers, poly[(4,4 bis(2 ethylhexyl)cyclopenta $\left[2,1 b: 3,4 b^{\prime}\right]$ dithiophene) 2,6 diyl al (2,1,3 benzothiadiazole) 4,7 diyl](PCPDTBT), and poly [(4,4' dioctyldithieno[3,2 $\left.b: 2^{\prime}, 3^{\prime} d\right]$ silol 2,6 diyl) alt (2,1,3 benzothiadiazole) 4,7 diyl)], (Si-PCPDTBT) were analyzed at the air-water interface forming a Langmuir monolayer. In order to form stable monolayers and to transfer to solid supports, amphiphilic molecules of stearic acid (SA) were mixed with them. For the pristine polymers, the floating monolayers were transferred onto solid substrates via the Langmuir-Schaefer (LS) technique. Surface pressure-area isotherms and compressibility modulus curves demonstrated that the SA incorporation to the polymers at the air-water interface modified the rheological properties of the Langmuir films, since the films became less compressible at higher pressures and there is clear conformational reorganization taking place at intermediary pressures. The UV-Vis absorption also depicted the changes on the overall film morphology by the shift on the maximum absorption bands, and along with cyclic voltammetry curves the absorption spectra made it possible to estimate the energy diagrams for the polymers. Photoconductivity effects were observed for all the sample, among which the pristine polymers fabricated by LS showed better results, suggesting that the organization provided by the Langmuir-Blodgett (LB) technique was not enough to overcome the insulating characteristic of the SA molecules in this specific configuration.
\end{abstract}

(C) 2018 Elsevier B.V. All rights reserved.

\section{Introduction}

The emergence of conducting polymers began the process of many studies, and the search for a comprehension of the electric conduction through the conjugation of chains, understanding that has enabled the creation of a new class of conductive polymers, the so-called lowbandgap polymers [1]. These materials have a low gap energy compared to existing polymers with gap energy above $1.5 \mathrm{eV}[2,3]$, besides a molecular structure with donor part and another acceptor of electrons, being a hybrid material with great potential for organic photonic devices [4,5].

Low-bandgap polymers are commonly used as the active layer of solar cells or field-effect transistors [6,7], however is still unwell explored by techniques that study and control its molecular stability that

\footnotetext{
* Corresponding authors.

E-mail addresses: vinijro@gmail.com (V.J. Rodrigues de Oliveira), hussein.awada@univ-pau.fr (H. Awada), hensan@uel.br (H. de Santana), roger.hiorns@univ-pau.fr (R.C. Hiorns),christine.lartigau-dagron@univ-pau.fr (C. Lartigau-Dagron), olivati@fct.unesp.br3 (C. de Almeida Olivati).
}

can contribute to a more favorable conformation of the film for its application in various devices $[8,9]$. The efficiency of organic devices based on low-bandgap polymers has been optimized with the use of polymers such as PCPDTBT and Si-PCPDTBT, due to its extensive optical absorption band widely used in the literature $[3,10]$.

The study of molecular monolayers and its organization onto liquid subphases has expanded due to diverse technological interests in the areas of engineering, chemistry, physics and biology. These interests stimulated a search for a better understanding of the researchers, with respect to the interaction of molecules in liquid subphases; and how the manipulation of these materials as conductive polymers can have their optical and electrical properties optimized and possibly applied in organic devices, such as: diodes, photodetectors or organic photovoltaic devices. Despite the vast theory and knowledge in the field of inorganic materials, there is still much to be discovered in relation to the processing of organic materials [11].

The optical and electronic properties of conductive polymer thin films, processed from solution, are normally associated with the way that the molecules are organized in the solid form. It is a determinant 
factor to control the organization process of the studied polymer, from a disordered state in solution to a state of better molecular ordering, as an attempt to achieve the most possible organized phase [12]. Hence, an effective way to study the assembly of conjugated polymers in an aqueous subphase is the Langmuir technique that allows the study of molecules under controlled and defined conditions enabling the analysis of mechanical properties, and molecular organization and interactions [13-15].

The assembled molecules over the water surface are called Langmuir films and they can be transferred onto solid substrates forming Langmuir-Blodgett (LB) and Langmuir-Schaefer (LS) films with controlled thicknesses, that are well-ordered and made using a small amount of material due to low concentration of the polymer solution required in these methods $[2,4,5]$.

Currently, analyzes by UV-Vis optical spectroscopy of low-bandgap polymers have been performed, making it possible to observe through the optical spectrum its extensive range of absorption and energy band around $900 \mathrm{~nm}[9,17,18]$. UV-Vis spectroscopy is also used to determine the optical bandgap, which value is usually close to the electronic gap reported $[9,17-19]$. As well electrochemical analysis, that provides the values of oxidation/reduction obtained by cyclic voltammetry (CV), are efficient in providing values that allow estimating the highest occupied molecular orbital (HOMO) and lowest unoccupied molecular orbital (LUMO). Due to these properties that can be monitored and improved using distinct deposition techniques, these materials are widely used in photoconductive applications [20,21].

The present study addresses the influence of the deposition technique and the addition of stearic acid to the polymeric system, and compares this impact in two different low-bandgap polymers. Thereby, isotherm and compressibility modulus analysis of the pristine polymers and their mixture with the fatty acid were investigated. For further examination, CV and UV-Vis absorption measurements were carried out to evaluate conformational and electronic features of the materials and estimate orbital levels during the compression of the lateral barriers that were moving. Moreover, through electrical characterization, the polymer solid films were tested for photoconductive effects.

\section{Experimental section}

\subsection{Materials}

The materials used in this study were the organic polymers poly [(4,4 bis( 2 ethylhexyl)cyclopenta $\left[2,1 b: 3,4 b^{\prime}\right]$ dithiophene) 2,6 diyl al (2,1,3 benzothiadiazole) 4,7 diyl] (PCPDTBT), $\mathrm{Mn}=18,000$

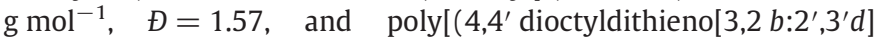
silol 2,6 diyl) (2,1,3 benzothiadiazole) 4,7 diyl)] (Si-PCPDTBT), $\mathrm{Mn}=$ $19,000 \mathrm{~g} \mathrm{~mol}^{-1}, Ð=2$, obtained by synthesis in accordance with literature $[22,23]$. The mass of the monomer of PCPDTBT and Si-PCPDTBT are 534.85 and $550.93 \mathrm{~g} \mathrm{~mol}^{-1}$ respectively. Stearic acid (SA) was purchased from Sigma-Aldrich and chloroform was acquired from Synth.

\subsection{Experimental procedures}

\subsubsection{Thin film fabrication}

Three solutions of the polymers with SA mixed in chloroform were prepared at different molar ratios (SA:PCPDTBT or SA:Si-PCPDTBT) of: $0: 1,2: 1$, and $4: 1$ (mmol) and concentrations of $0.2,0.55$ and $0.84 \mathrm{mg} /$ $\mathrm{ml}$ respectively. The solutions were sonicated for 30 to $45 \mathrm{~min}$. A Langmuir trough (KSV 5000 model) was used for the analysis of the molecules in aqueous subphase and to fabricate the solid films via vertical dipping (LB) and horizontal lifting (LS). The subphase was filled with ultrapure water from a Millipore water purifier for particulate retention with a filter of $0.22 \mathrm{~nm}$, producing water at $25{ }^{\circ} \mathrm{C}$ with resistivity of $18.2 \mathrm{M} \Omega \cdot \mathrm{cm}$ and $\mathrm{pH}=6.4$. The solutions according to the information in the Table 1 were spread on the ultrapure water surface contained in the Langmuir trough, and waited 15 min for solvent evaporation. The monolayers formed on the air-water interface were analyzed by surface
Table 1

LB and LS deposition parameters of PCPDTBT and Si-PCPDTBT low-bandgap polymers.

\begin{tabular}{lllllll}
\hline & PCPDTBT & \multicolumn{5}{c}{ Si-PCPDTBT } \\
\hline Proportion between SA and polymer $(\mathrm{mmol})$ & $0: 1$ & $2: 1$ & $4: 1$ & $0: 1$ & $2: 1$ & $4: 1$ \\
Deposition pressure $(\mathrm{mN} / \mathrm{m})$ & 25 & 30 & 30 & 25 & 40 & 40 \\
Scattered volume $(\mu \mathrm{L})$ & 500 & 200 & 175 & 500 & 200 & 175 \\
Layers & 30 & 35 & 51 & 30 & 35 & 35 \\
Average transfer ratio & - & 0.8 & 0.8 & & 0.8 & 0.85 \\
Speed up stroke $(\mathrm{mm} / \mathrm{min})$ & - & 9 & 9 & & 8 & 9 \\
Speed down stroke & - & 8 & 8 & & 8 & 8 \\
\hline
\end{tabular}

pressure versus area per polymeric unit ( $\pi-\mathrm{A})$ isotherms at $23^{\circ} \mathrm{C}$, using a Wilhelmy sensor that detects the surface tension changes on the aqueous surface during the compression owing to the lateral barriers that were moving at a speed of $15 \mathrm{~cm}^{2} / \mathrm{min}$. To obtain the $\pi-A$ isotherms, only information of SA (molecular weight and concentration) was added into the Langmuir trough software, emphasizing the influence of SA molecules in the mixed polymeric chloroform solutions.

After identifying, through $\pi-A$ isotherms and their experimental repetitions, the surface pressure in which the molecules that form the Langmuir films present better organization, this chosen pressure was then kept constant, and it was performed the transfer of these monolayers onto glass substrates with gold interdigitated electrodes (IDE) that were fabricated using photolithography as detailed elsewhere [24]. The deposition was accomplished by two ways: the LB technique where the substrates moved up and down successively in a vertical position in relation to the interface, and the deposition parameters were controlled as seen on Table 1; and using the LS technique in which the substrate horizontally touches the surface containing the Langmuir film and it is lifted afterwards. For the latter method, only the pressure can be controlled by the software. The two methods offer films in different architectures, however both produce nanostructured films [25,26].

\subsection{Characterization of the thin films}

Drop-cast films of PCPDTBT and Si-PCPDTBT polymers were prepared to perform CV measurements. The substrate used for CV analysis was platinum. The polymer film formed on the platinum was subjected to electrochemical impedance, with a scan rate of $50 \mathrm{mV} / \mathrm{s}$ in the electrochemical cell. The CV curves were obtained using an Autolab Potentiostat/Galvanostat PGSTAT $302 \mathrm{~N}$. The electrochemical cell was composed of $0.1 \mathrm{~mol}$ of lithium perchlorate solution $\left(\mathrm{LiClO}_{4}\right)$ in Acetonitrile (ACN). The polymer HOMO level was estimated by the oxidation peak from the CV characterization.

The polymers in solution and transferred onto transparent glass substrates were analyzed by optical characterization in a Cary 100 UV-Vis spectrophotometer, making it possible to determine the optical bandgap energy of the materials. Additionally these measurements helped to investigate some properties such as organization and conjugation length, for the polymer solutions and the thin films.

The photoconductivity capability of the SA:polymer films were investigated by direct current (DC) electrical measurements using the equipment Keysight voltage source model B2901A and the Oriel Solar Simulator VERASOL LED (AM 1.5G) as the light source. The materials transferred onto the IDE substrates were kept under dark prior to data acquisition, then it was applied a constant voltage of $5 \mathrm{~V}$ and allowed the current to stabilize, the sample was illuminated, and this system was maintained until the photocurrent presented a steady trend, and after this process the light was turned off.

\section{Results and discussion}

\subsection{Langmuir isotherms $(\pi-A)$}

Fig. 1 shows the surface pressure isotherms by area ( $\pi-A)$ of the lowbandgap polymers a) PCPDTBT and b) Si-PCPDTBT, both pristine and 

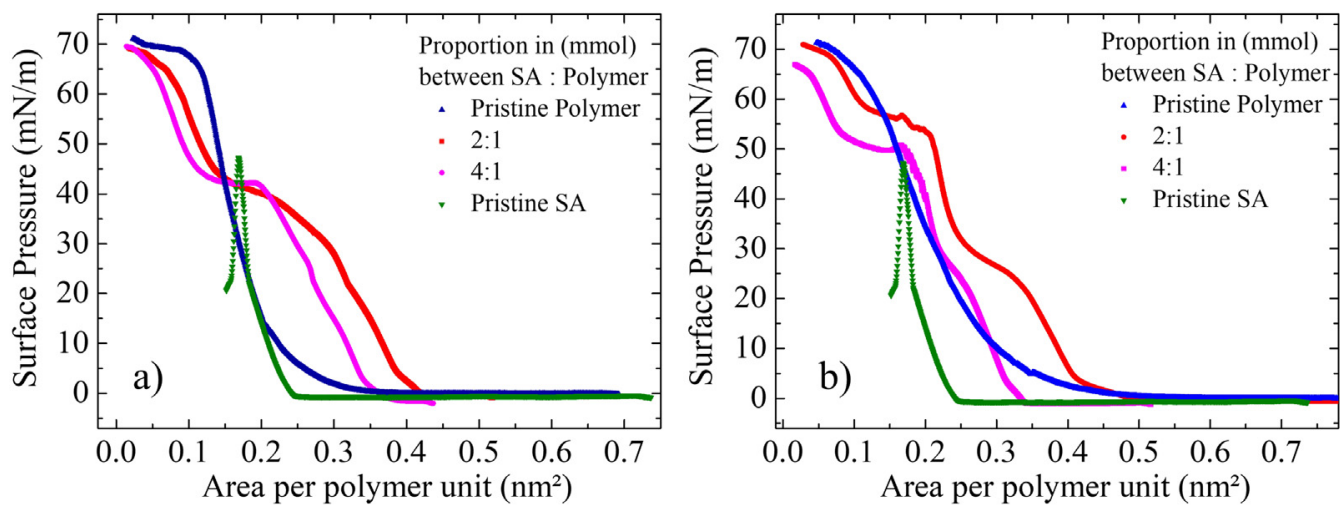

Fig. 1. Surface pressure isotherms by area ( $\pi-A)$ of the polymers, a) PCPDTBT and b) Si-PCPDTBT, pristine and mixed with SA.

mixed with SA. The collapse pressure of the pristine SA molecule takes place at around $50 \mathrm{mN} / \mathrm{m}$, and an observed collapse pressure for each of the pristine polymers (PCPDTBT and Si-PCPDTBT) is about $70 \mathrm{mN} /$ $\mathrm{m}$. When analyzing the $(\pi-\mathrm{A})$ isotherms, containing the curves for the polymers, the SA and their mixtures, inflections in the curves of mixed solutions can be observed, inflections that would be indicative of collapse occurring [27]. This can be clearly seen at pressures around 50 and $70 \mathrm{mN} / \mathrm{m}$ for the $2: 1$ and $4: 1$ proportions of SA:Si-PCPDTBT, and pressures around $40 \mathrm{mN} / \mathrm{m}$ and $70 \mathrm{mN} / \mathrm{m}$ for the $2: 1$ and $4: 1$ proportions of SA:PCPDTBT. Additionally for the SA:Si-PCPDTBT films, there is another change in slope around $30 \mathrm{mN} / \mathrm{m}$, which suggests another molecular rearrangement of the materials on the trough that did not occur for the other polymer [14].

However, an analysis using the excess of the average area per molecule $\left(A_{E}\right)[28]$, using the Eq. (1).

$A_{E}=A_{12}-\left(X_{1} A_{1}+X_{2} A_{2}\right)$

where $A_{12}$ is the average molecular area in the two-component film, $X_{1}$ and $X_{2}$ are the molar fractions of two components, and $A_{1}$ and $A_{2}$ are the molecular areas of two single-component films at the same $\pi$.

The quantitative analysis using the additivity rule performed in $\pi=$ $30 \mathrm{mN} / \mathrm{m}$ for PCPDTBT and $\pi=40 \mathrm{mN} / \mathrm{m}$ for Si-PCPDTBT applies to the mixtures, and in order to recognize whether we are dealing with an ideal mixture of phases or not. With $A_{E}$ of PCPDTBT 2:1 and 4:1 of 0.203 and $0.145 \mathrm{~nm}^{2}$ respectively; for the Si-PCPDTBT $2: 1$ and $4: 1$ of -0.025 and $0.025 \mathrm{~nm}^{2}$. This method uses Gibbs free energy for mixtures $\Delta G_{\text {mix }}^{\text {exc }}$ (Eq. (2)), which represents the energy gain related to the mixing process for the components in a system.

$\Delta G_{\text {mix }}^{\text {exc }}=\int_{0}^{\pi}\left(A_{12}-X_{1} A_{1}-X_{2} A_{2}\right) d \pi$

The observed Gibbs free energy excess deviations did not present null values, which indicates a miscibility condition and non-ideal standard behavior of the materials during compression in the analyzed subphase, since the analyzes were made at the deposition pressures of the materials $[28,29]$. Positive values of $\Delta \mathrm{G}$ indicate that the mixing process is not thermodynamically stable, and that a two-component monolayer indicates that the interactions between them are weaker than the interactions of the pure components themselves $[13,30]$.

The area per polymer unit for the SA in the isotherms obtained experimentally in this work reached an area of around $0.211 \mathrm{~nm}^{2}$, being in agreement with the literature [14,24]. The area per molecule for the pristine polymer unit isotherms is 0.243 and $0.315 \mathrm{~nm}^{2}$ for PCPDTBT and Si-PCPDTBT respectively. Despite the low miscibility between materials, the SA showed great influence on the conformation of the molecules in the trough; thus altering the overall features of the graphs, and the areas found for the molecules (Fig. 1a) and b)). For the SA:PCPDTBT
Langmuir films in Fig. 1a), the average area per polymeric unit in their condensed phase is 0.52 and $0.395 \mathrm{~nm}^{2}$ for the proportions in (mmol) of 2:1 and 4:1, respectively. And for SA:Si-PCPDTBT in Fig. 1b), with the same proportions of SA and polymer, the area reached per molecule is about 0.278 and $0.269 \mathrm{~nm}^{2}$. These results demonstrate that the presence of the SA significantly changes the isotherms attained for the pristine polymers, whereas both polymers mixtures presented great shift of the isotherms when compared to the pristine SA curve, indicating that the polymer and the SA units are sharing the water interface space rather than laying one on the top of the other. The films of the 2:1 proportions revealed a larger area for the polymers when compared to that at $4: 1$, indicating that the interaction between polymer and SA is reasonably changed as their ratio changes [14,24].

Generally, a buffer solution is used as the subphase in order to keep the pH constant, which allows a better analysis of the Langmuir monolayer. However, in this study, one of the goals was to transfer the polymers onto solid substrates and characterize them, thus the insertion of new additional molecular species could affect the solid thin films formation. Anyhow, experiments utilizing buffer solution were carried out and there were no substantial differences on the obtained results.

The addition of SA in a polymer mixture in aqueous subphase is known for serving as molecular spacer for the stabilization of the polymer at the air-water interface which decreases the polymer aggregates formation. It was not possible to deposit the pristine polymers by the LB technique, due to rigidity of the monolayer, a fact that made deposition by LS necessary. The deposition parameters used are shown in Table 1. This behavior is similar to what happens to other pristine polymers when deposited by LB [24,27].

This study of the SA:polymer films, showed that this SA addition to the polymer corroborates the fact that some pristine polymers are not suitable for LB deposition, making it necessary the incorporation of lipid molecules such as SA, to then produce high quality thin films [31]. A clear change on slope of the curves for surface pressure values close to $35 \mathrm{mN} / \mathrm{m}$ (Fig. 1), can be attributed to a molecular accommodation of the polymer over the molecules which are laying on the aqueous subphase while compression is occurring [31].

3.2. $C s^{-1}$ vs A curves

The compressibility of the monolayers was analyzed in order to characterize and understand the specifics of the transition from liquidexpanded (LE) to liquid-condensed (LC) regimes, where this transition is typically characterized by the abrupt decrease of the molecular area during the compression of the molecules in the Langmuir trough [32]. The results are given through plots of the compressibility modulus $\left(\mathrm{Cs}^{-1}\right)$ versus the mean molecular area, and $\mathrm{Cs}^{-1}$ is given by the Eq. (3):

$C s^{-1}=-A\left(\frac{\delta \pi}{\delta A}\right)_{T}$ 


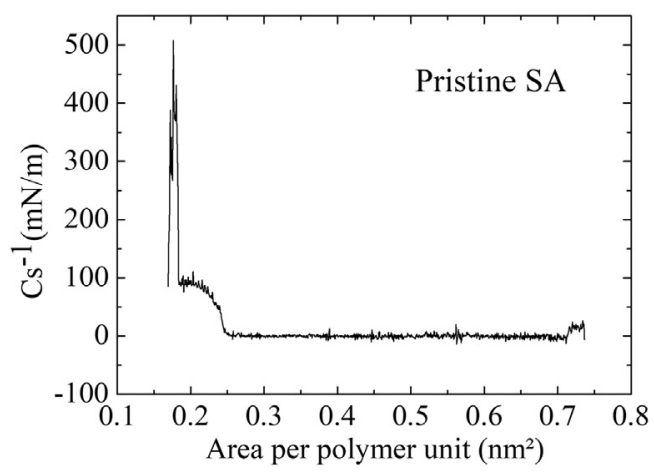

Fig. 2. Area compressibility modulus ( $\mathrm{Cs}^{-1}$ vs. A) of pristine stearic acid (SA).

where A represents the area per molecule, $\pi$ is the surface pressure. Thus, $\mathrm{Cs}^{-1}$ has unit of surface pressure, $\mathrm{mN} / \mathrm{m}$. The curves of $\mathrm{Cs}^{-1}$ can be plotted as a function of surface pressure or the area per polymeric unit, allowing us to determine the surface pressure and certain transition regions of the studied molecules, making a relation of $\mathrm{Cs}^{-1}$ and tabulated values $[13,32]$.

Fig. 2 presents the compressibility modulus for the SA Langmuir films and this graph has a standard behavior for molecular compression of SA in the trough, since SA is one of the ideal amphiphilic molecules for Langmuir studies, with the changes of the states well defined [14,16,24]. It can be observed that during the beginning of the compression (larger areas), the $\mathrm{C}_{\mathrm{s}}^{-1}$ stays around zero, meaning that there is no interaction of the molecules in the aqueous subphase, indicating that this is the gas phase. The compressibility modulus $\left(\mathrm{Cs}^{-1}\right)$ begins to increase (around $12 \mathrm{mN} / \mathrm{m}$ ) as the surface pressure increases, because the molecular interactions begin to appear, pointing to phase changes, followed by a reorganization that leads to a transition phase corresponding to the beginning of the condensed phase according to the Harkin's classification (at approximately $250 \mathrm{mN} / \mathrm{m}$ ) [13].

The compressibility curves for the pristine polymers are displayed in Fig. 3a) and d), and the peak points correspond to the state where the monolayers reveal maximum compressibility [32]. Upon compression a rise on the compressibility of the two polymers is obtained, suggesting that the films suffered a transition from a gaseous phase $\left(\mathrm{Cs}^{-1} \sim 0 \mathrm{mN} / \mathrm{m}\right)$ to a phase more condensed [33].The maxima $\mathrm{Cs}^{-1}$ calculated for each polymer was approximately $130 \mathrm{mN} / \mathrm{m}$ corresponding to a liquid condensed regime [13]. Although both polymers are in the same regime, the PCPDTBT Langmuir film seems to be slightly more rigid than those of Si-PCPDTBT, once the $\mathrm{Cs}^{-1}$ for PCPDTBT reach high values more consistently.

Using the compression method, the LE-LC phase transition of monolayers containing the polymers with different amounts of SA has also been evaluated. Fig. 3b), c), e) and f) display the curves for the SA:polymer films. The $\mathrm{Cs}^{-1}$ maxima peaks for SA:PCPDTBT films stayed around the same values as for the pristine polymers $(130 \mathrm{mN} / \mathrm{m})$, while for the SA:Si-PCPDTBT films the maximum $\mathrm{Cs}^{-1}$ has been shifted to over 200 $\mathrm{mN} / \mathrm{m}$ for the $2: 1$ samples and nearly $300 \mathrm{mN} / \mathrm{m}$ for the $4: 1$ samples. This outcome indicates a more densely packed monolayer of the SiPCPDTBT mixed films in comparison to its pristine form and to all the PCPDTBT films, however there is no phase change since this result still relies on the LC phase range [34]. The $\mathrm{Cs}^{-1}$ values of the mixed monolayers reveal several minima and maxima, and this is related to the changes in the slope of the corresponding isotherms. These inflections reveal the phases and the transitions between them, since there are only the gaseous-LE-LC transitions on the isotherms, result inferred by the compressibility modulus attained, the other slopes are due to conformational rearrangements on the films lying over the water subphase.

The pristine polymer and SA showed a single peak of maximum compressibility, however the mixed proportions presented several $\mathrm{C}_{s}^{-1}$ peaks, this fact associated with presence of significant changes in slope of $\pi-A$ isotherms and steep minima in the $C_{s}^{-1}$-A curves imply that the materials on the mixed films are immiscible with one another at the air-water interface [35], with a multiple-phase system with probable weak interaction between them giving origin to the appearance of the collapse pressure of the polymer and the SA.

\subsection{UV-Vis spectroscopy data}

The degree of organization and how molecules are differently organized in solution and in solid state can be observed by means of the optical absorption spectra, whereas a comparison of the polymers in solution and deposited onto transparent solid glass substrates is performed. The UV-Vis absorption spectra of PCPDTBT can be visualized in Fig. 4a). For the absorption solution spectra, an absorption band
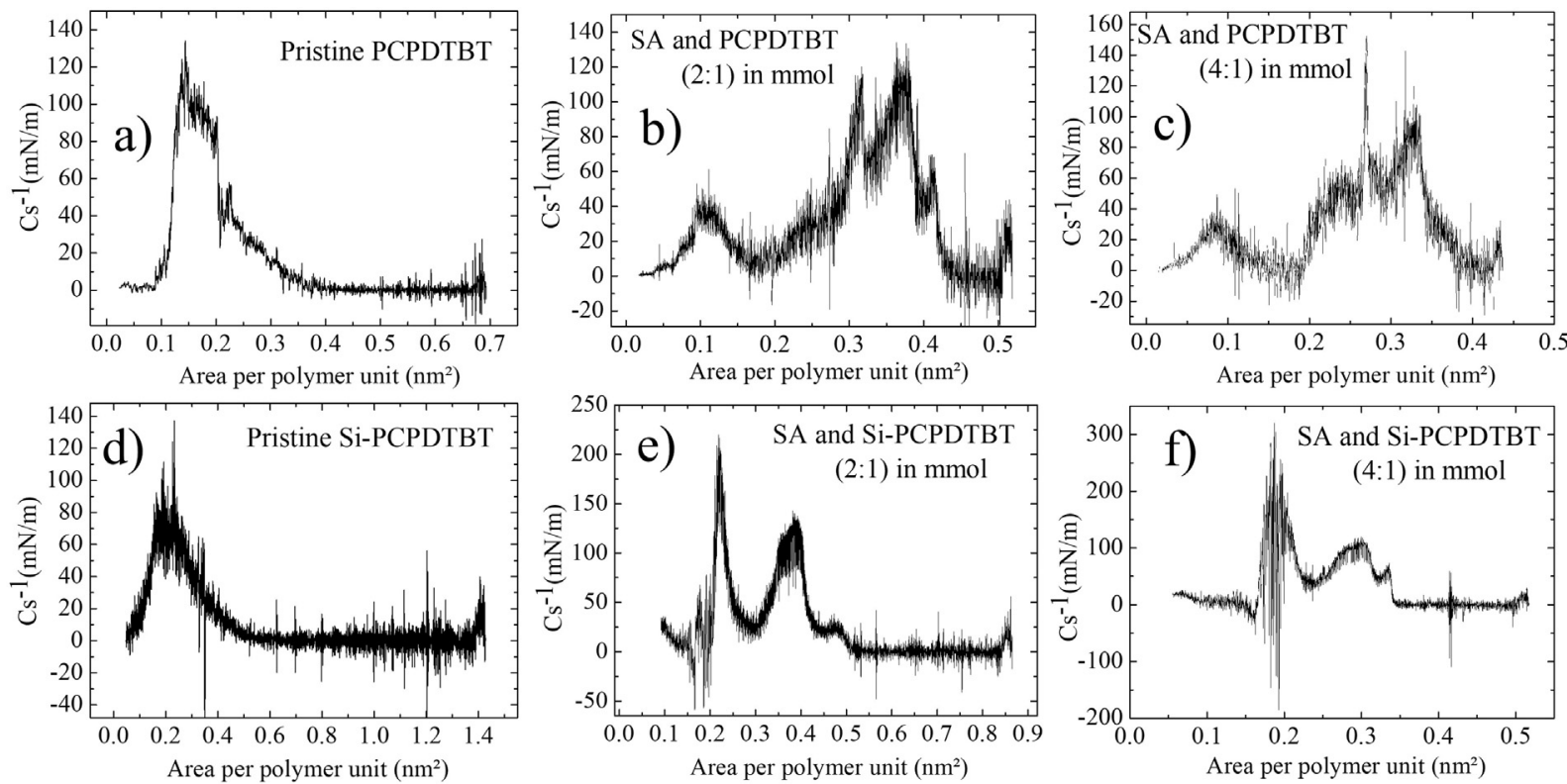

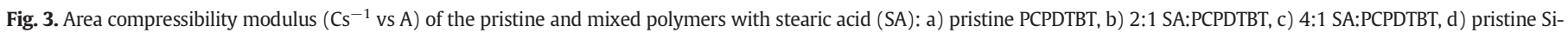
PCPDTBT, e) 2:1 SA:Si-PCPDTBT and f) 4:1 SA:Si-PCPDTBT. 

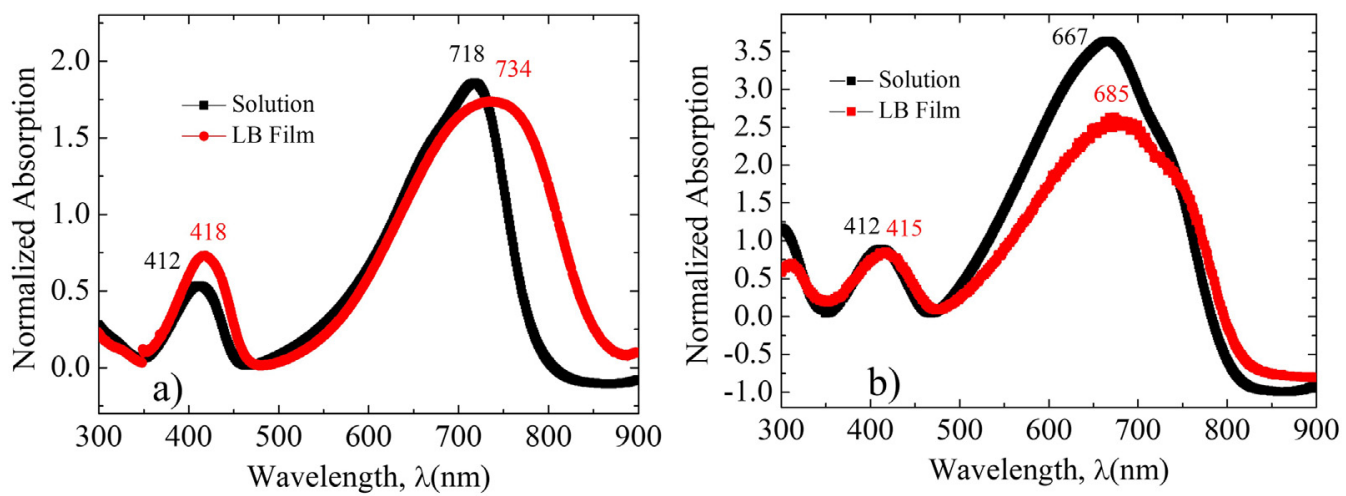

Fig. 4. UV-Vis absorption spectrum for mixed 2:1 SA:polymer of a) PCPDTBT and b) Si-PCPDTBT, both in chloroform solution and LB film (35 layers).

with first peak at $412 \mathrm{~nm}$ and then at $718 \mathrm{~nm}$ can be observed in Fig. 4a), when compared to films processed by LB in proportion 2:1 SA:polymer, the thin film which was on the solid substrate showed greater absorption band shifts at 418 and $734 \mathrm{~nm}$. This same shift of the bands happened for the solid thin films (in proportion 2:1 SA:polymer) and it can be observed in (Fig. 4b), the first absorption peak of the SiPCPDTBT solution is centered at 412 and the second at $667 \mathrm{~nm}$, while for the solid film, it also occurs a redshift to 415 and $685 \mathrm{~nm}$. These redshifts are expected since the conjugation length and overall organization are usually higher when passed to a solid substrate [36].

Figs. 5 and 6 present the UV-Vis absorption spectra pointing out the growth of Langmuir layers onto solid substrates for pristine and mixed films of SA:PCPDTBT and SA:Si-PCPDTBT, respectively. Measurements and spectrum analysis were performed to control layer growth and reproducibility. Analyzing the results obtained, one can observe a satisfactory linear growth, showing that the processing technique transfers roughly the same amount of material per transferred layer. This type of analysis consists of an important tool to further control thickness of future devices.

Regarding the absorption spectra of the two polymers PCPDTBT and Si-PCPDTBT analyzed and their mixtures containing different proportions, it was possible to highlight two peaks in the spectra, the highest energy band with values close to $400 \mathrm{~nm}$, and the lower energy band around the $700 \mathrm{~nm}$. These two prominent peaks in the spectra can be related to the two monomers that constitute the polymer, being the first peak related to the CPDT (4,4 bis (2 ethylhexyl) 2,6 bis (trimethylstannyl) $4 \mathrm{H}$ cyclopenta $\left[2,1 \mathrm{~b}: 3,4 \mathrm{~b}^{\prime}\right]$ dithiophene) part, while the second peak is related to the BT (4,7 dibromo 2,1,3 benzothiadiazole) group, and also with intermolecular charge transfer (ICT) between CPDT and BT $[4,37,38]$. Where for both blends and materials in their pristine form, presented a greater band shift for the smaller proportions of SA, showing that a minimum quantity can improve the film organization, possibly improving its electrical features [27].

\subsection{Electrochemical and optical spectroscopy}

\subsubsection{Cyclic voltammetry}

The results obtained by $\mathrm{CV}$, allowed estimating the HOMO and LUMO values of the processed materials, in which the orbitals are related to the electronic affinity and the ionization potential, enabling an estimation of these values [2].
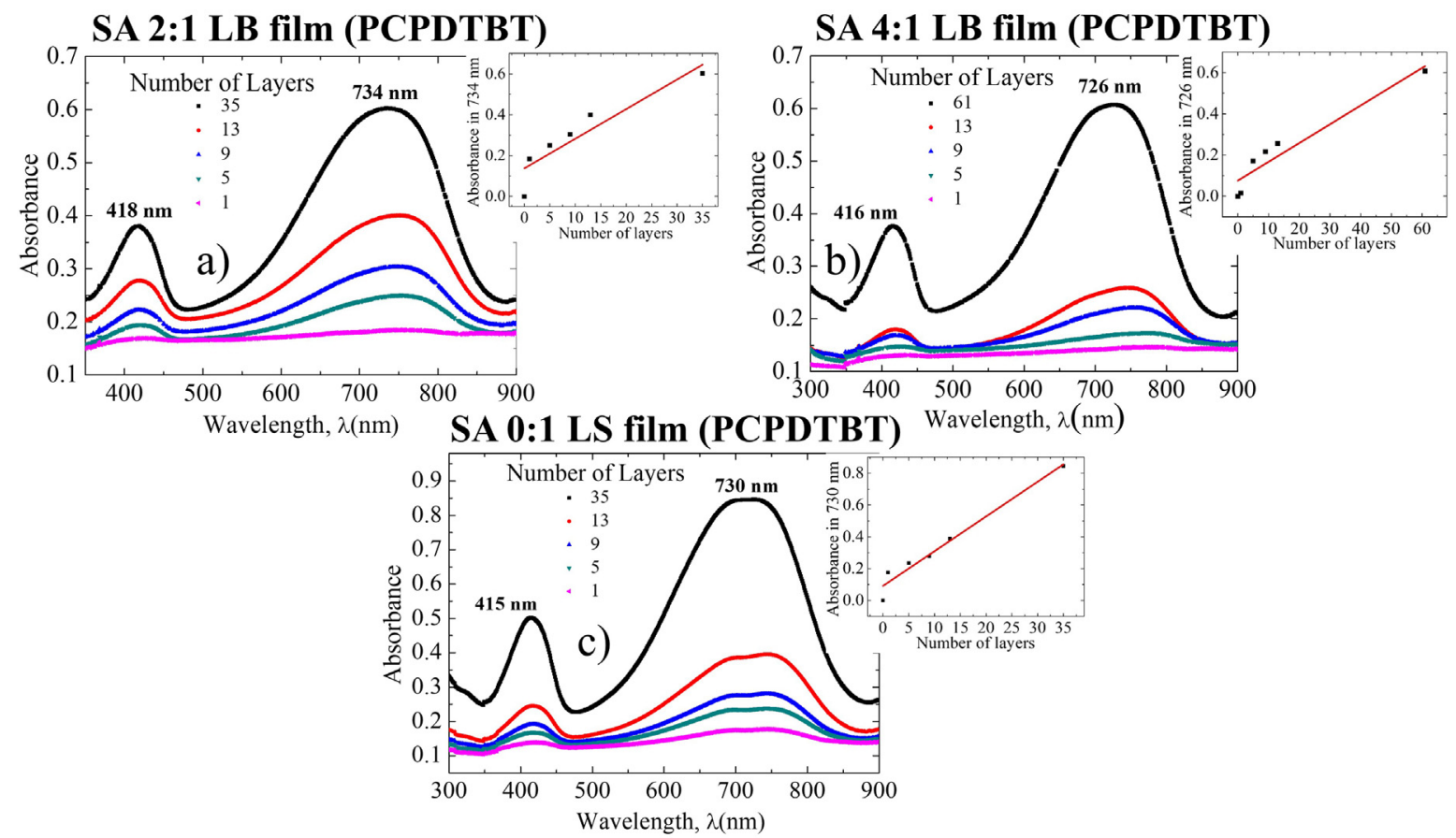

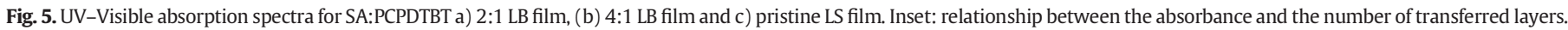



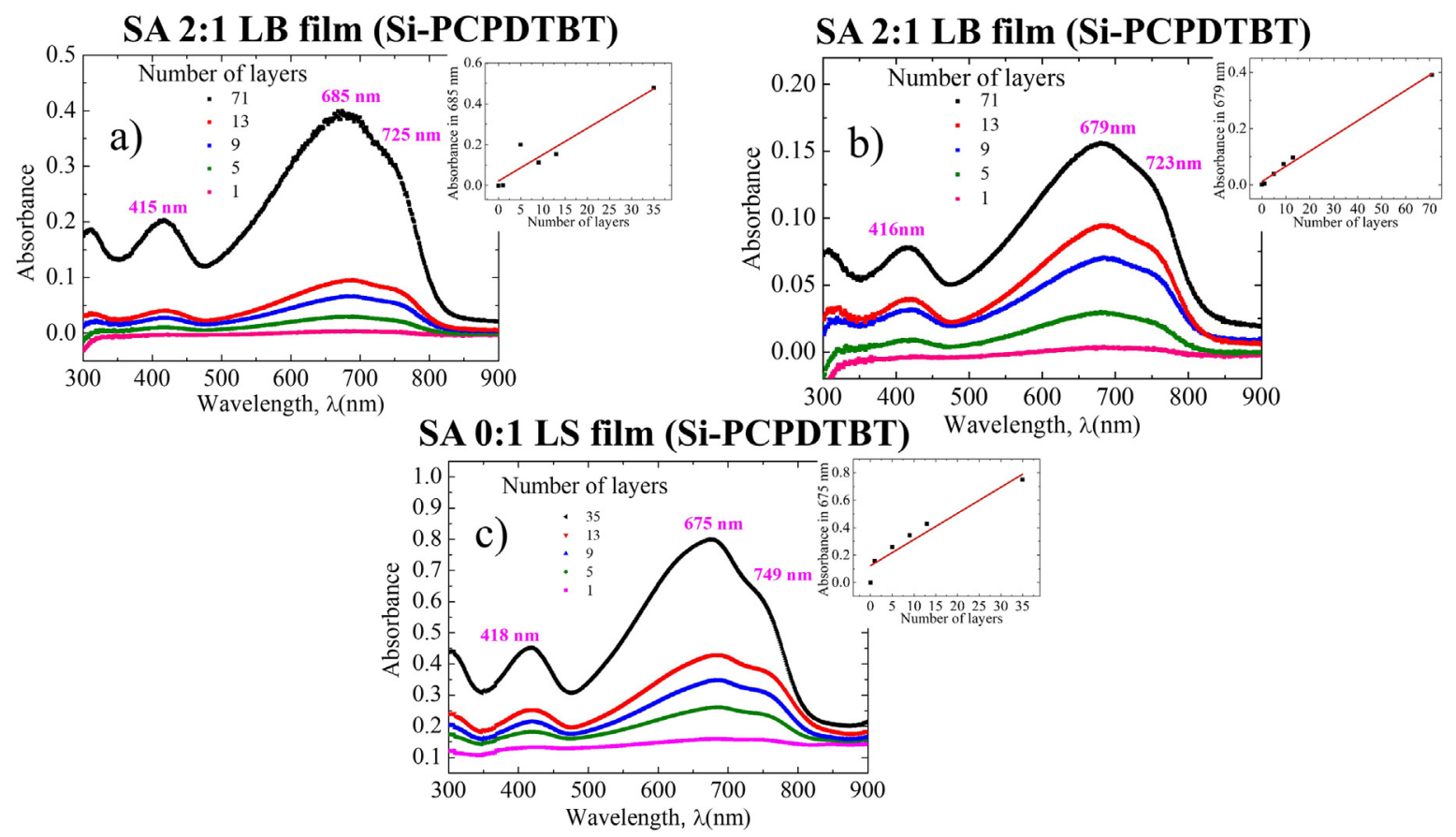

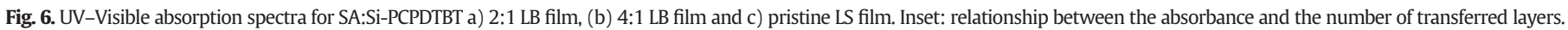

The CV curves obtained for the polymers PCPDTBT and Si-PCPDTBT (Fig. 7) deposited onto solid substrates by the drop-casting technique, presented a single oxidation peak which can be acquired by the onset of this peak ( $E_{\text {ox onset }}^{\prime}$ [2]. Generally, CVs of polymers with similar structures to the ones from this study (PCPDTBT and Si-PCPDTBT) present rather indefinite curves, making it difficult to define its oxidation and reduction peaks [18], and from the results presented here only the oxidation peak of the samples can be estimated. Some possible causes for this behavior are the specific polymer characteristics, such as the size of its conjugation length and even its molecular weight $[2,18]$.

For the electrochemical measurements carried out, a correction value was required for the specific electrode used, this value $(4.43 \mathrm{eV})$ has been inserted in the following Eq. (4) aiming the determination of the HOMO energy level ( $\left.E_{\text {HOMO }}\right)$, whereas this correction value is the reference potential for the electrode used with the measurement performed in non-inert atmosphere. The values of the oxidation potential onset $\left(E_{\text {ox }}^{\prime}\right)$, gap energy $\left(E_{\text {gap }}\right)$, and the LUMO energy level $\left(E_{\text {LUMO }}\right)$ are shown in Table 2 where

$E_{\text {Hомо }}=-\mathrm{e}\left(E_{\text {ox onset }}^{\prime} \mathrm{vs} \mathrm{Ag} / \mathrm{AgCl}+4.43\right) \mathrm{eV}$
The HOMO energy obtained for the PCPDTBT and Si-PCPDTBT polymers were around 4.8 and $4.78 \mathrm{eV}$, respectively (Table 2). Due to the impossibility of estimating the electronic affinity peak of the anodic potential, it was not possible to establish the reduction peak in order to determine the LUMO. Thus, it was necessary to use UV-Vis spectral data, ie the onset wavelength and through Eq. (5) the optical $E_{\text {gap. }}$.

$E_{\lambda}(\lambda)=h v=h(c / \lambda)$

where $h$ is the Planck constant and $c$ is the speed of light.

The calculated optical $E_{\mathrm{Gap}}$ can be used, since it is close to the electronic $E_{\text {Gap }}$ of conjugated polymers $[39,40]$. The $E_{\text {Gap }}$ calculated by Eq.

Table 2

Values of $\mathrm{E}_{\mathrm{ox}}(\mathrm{V}), \mathrm{E}_{\mathrm{Gap}}(\mathrm{eV}), \mathrm{E}_{\mathrm{HO} O}(\mathrm{eV}), \mathrm{E}_{\mathrm{LUMO}}(\mathrm{eV})$ obtained by cyclic voltammetry and UV-Vis optical absorption.

\begin{tabular}{llllll}
\hline Polymer & Sweep & $E_{\text {ох }}(\mathrm{V})$ & $E_{\text {Gap }}(\mathrm{eV})$ & $E_{\text {номо }}(\mathrm{eV})$ & $E_{\text {LUмо }}$ \\
\hline PCPDTBT & $50 \mathrm{mV} / \mathrm{s}$ & 0.37 & 1.44 & 4.8 & 3.36 \\
Si-PCРDTBT & & 0.35 & 1.5 & 4.78 & 3.28 \\
\hline
\end{tabular}
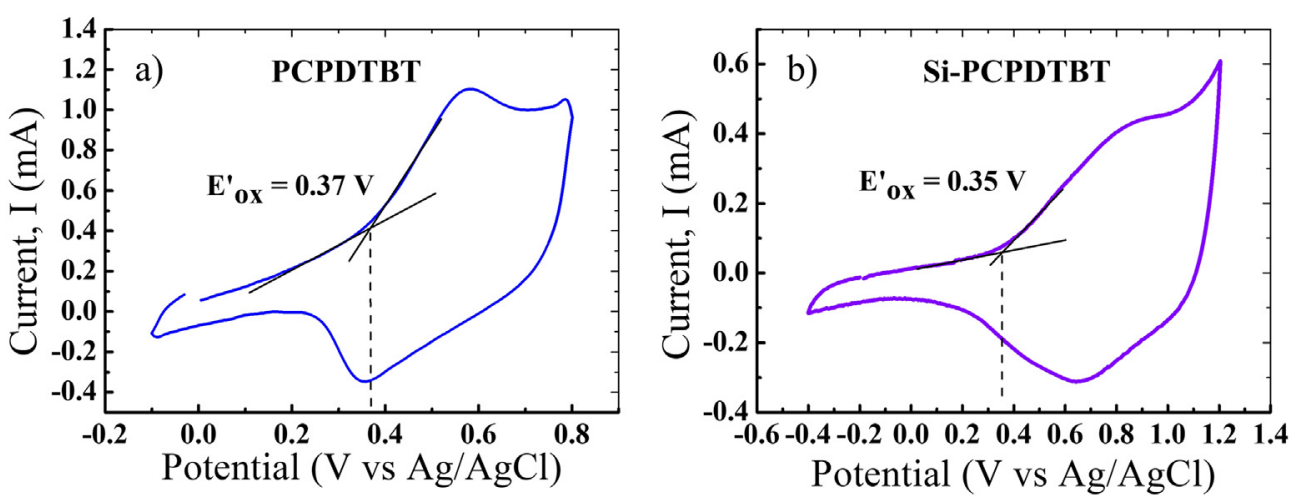

Fig. 7. Cyclic voltammograms obtained for a) PCPDTBT and b) Si-PCPDTBT deposited as drop-cast films. 
(5) is related by an intersection at the end of the UV-Vis band (Figs. 5 and 6), being the wavelength related to the optical $E_{\text {Gap }}$ [23]. Using the equation: $E_{\mathrm{LUMO}}=E_{\mathrm{HOMO}}-E_{\mathrm{Gap}}$, the LUMO level can be calculated. Thereby, it is possible to estimate the energetic levels of the polymer, once one can consider the process of charge transfer as reversible during CV $[2,41]$.

The results obtained for $E_{\text {Gap }}$ were around 1.43 and $1.49 \mathrm{eV}$, and these values are slightly lower than those observed for these materials when processed by other techniques $[4,18,37]$, and are close to those observed for these materials when processed by drop-casting $[42,43]$. This difference can be related to the conformation of the Langmuir films and could work as a way of optimizing the organic devices performances $[9,18]$.

The slight difference between the results obtained for Si-PCPDTBT, which presented higher value of $E_{\mathrm{Gap}}$, can be attributed to the silicon atom in its structure, since it is the only difference among these two low-bandgap polymers. The silicon atom size can be responsible for modifying significantly the solid films morphology and therefore their optical and electronic properties [44].

\subsection{Electrical characterization}

\subsubsection{Photoconductivity}

Fig. 8 shows the characteristic responses in the dark and under illumination for the LB and LS films of PCPDTBT and Si-PCPDTBT deposited onto interdigitated electrodes. The results stress the photoconductivity effect of these low-bandgap polymers regardless of the thin film deposition technique. The electrical response of all samples proved to be immediate when they are under illumination. The most sensitive films (that exhibit higher response to light) are the pristine PCPDTBT and Si-PCPDTBT films.

One hypothesis for such outcome is the insulating characteristic of the SA molecules which could cause a decrease of the charges generated after the photon capture, as well as the hindering of the charge mobility due to possible traps in the structure [45]. Another hypothesis is that the mixed film formed in the Langmuir trough forms a bilayer, in which the under layer is formed predominantly by insulating SA while the upper layer is formed mostly by the low-bandgap polymer. When the sample is illuminated vertically, the polymer absorbs the photons and conducts the charges generated to the electrode below. During this process the charger must pass through the insulating layer of SA, modifying the efficiency of the devices and possibly decreasing the efficiency of this device [46]. However, the addition of SA molecules allows the LB deposition generating thinner and more organized films [24,27,47].

\section{Conclusions}

In this paper we analyzed the features of two low-bandgap polymers (PCPDTBT and Si-PCPDTBT) and their interaction with a standard amphiphilic molecule (SA), forming a Langmuir monolayer at the surface of an aqueous subphase. These Langmuir monolayers were deposited onto solid substrates by LB and LS techniques in order to perform optical and electrical measurements. Through the surface pressure isotherms and the compressibility modulus curves was possible to obtain information concerning the behavior of the pristine and mixed low-bandgap polymers. The outcome showed that the films attain the liquid condensed phase, and that the silicon atom on the Si-PCPDTBT affected considerably the interaction with the fatty acid (SA). The SA enabled the fabrication of LB films with good transfer ratios, and more organized films as shown by the UV-Vis absorption spectra. The energy diagram for the pristine polymers was obtained by the CV graphs of the polymeric films deposited by drop-cast. These low-bandgap polymers presented photoconductivity effects, and the interaction among polymer and SA as well the deposition technique played an important role on the result. Hence, this work offers a comparison study between two similar low-bandgap polymers, from their aspects at air-water interface in pristine and mixed form, besides it shows the possibility to fabricate solid thin films and devices using techniques not commonly used for low-bandgap polymers.

\section{Acknowledgment}

The authors are grateful for the financial support of the Brazilian agencies CAPES, CAPES/COFECUB (NANOSOL, Ch 748 26805WM), FAPESP (Grant 2010/20094-1), INEO/CNPq and LNNano (CNPEM-Brazil) for providing gold interdigitated electrodes (project LMF 16557).
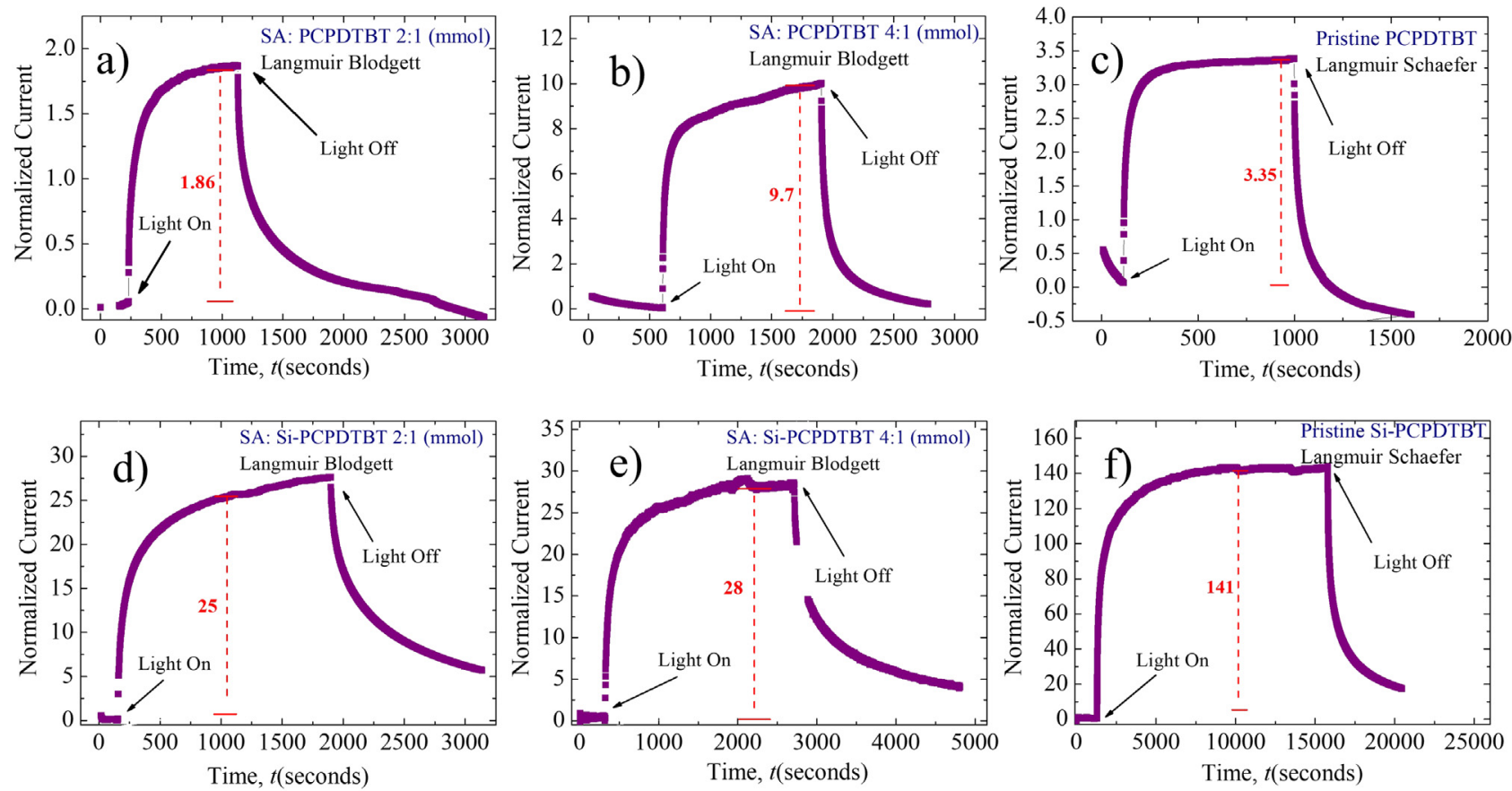

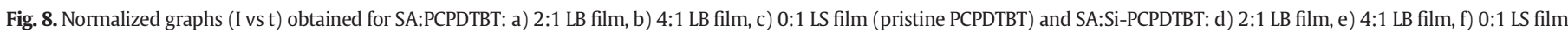
(pristine Si-PCPDTBT). All films were deposited onto interdigitated electrodes to perform the photoconductivity measurements. 


\section{References}

[1] G. Li, W. Chang, Y. Yang, Low-bandgap conjugated polymers enabling solution-processable tandem solar cells, Nat. Rev. Mater. 2 (2017) 1-13, https://doi.org/10.1038/ natrevmats.2017.43.

[2] M.L. Braunger, A. Barros, M. Ferreira, C.A. Olivati, Electrochimica Acta Electrical and Electrochemical Measurements in Nanostructured Films of Polythiophene Derivatives, 165, 2015 1-6.

[3] C. Scharsich, F.S.U. Fischer, K. Wilma, R. Hildner, S. Ludwigs, A. Köhler, Revealing structure formation in PCPDTBT by optical spectroscopy, J. Polym. Sci. B Polym. Phys. 53 (2015) 1416-1430, https://doi.org/10.1002/polb.23780.

[4] S.L. Fronk, M. Wang, M.J. Ford, J.E. Coughlin, C.-K. Mai, G.C. Bazan, Effect of chiral 2 ethylhexyl side chains on chiroptical properties of the narrow bandgap conjugated polymers PCPDTBT and PCDTPT, Chem. Sci. 7 (2016) 5313-5321, https:// doi.org/10.1039/C6SC00908E.

[5] J. Mei, K.R. Graham, R. Stalder, S.P. Tiwari, H. Cheun, J. Shim, M. Yoshio, C. Nuckolls, B. Kippelen, R.K. Castellano, J.R. Reynolds, Self-assembled Amphiphilic Diketopyrrolopyrrole-based Oligothiophenes for Field-effect Transistors and Solar Cells, 2011 2285-2288.

[6] W.P. Mengmeng Li, Felix Hinkel, Klaus Müllen, Self-assembly and charge carrier transport of solution-processed conjugated polymer monolayers on dielectric surfaces with controlled sub-nanometer roughness, Nanoscale 6 (2016) 9211-9216 https://doi.org/10.1039/C6NR01082B.

[7] J. Yu, Y. Zheng, J. Huang, Towards high performance organic photovoltaic cells: a review of recent development in organic photovoltaics, Polymers 6 (2014) 2473-2509, https://doi.org/10.3390/polym6092473.

[8] N. Fröhlich, R. Tautz, E. Da Como, C. Wiebeler, G. Soavi, I. Dumsch, Charge photogeneration in donor-acceptor conjugated materials: influence of excess excitation energy and chain length, Am. Chem. Soc. 135 (2013) 4282-4290, https://doi. org/10.1021/ja309252a.

[9] S. Santosh, K. Raavi, G. Grancini, J. Yin, C. Soci, A. Petrozza, H.J. Snaith, G. Lanzani, Ultrafast charge photogeneration in low band-gap semiconducting polymer based solid-state dye sensitized solar cell (sDSC), Light. Energy Environ. (2014), PTu4B.5. https://doi.org/10.1364/PV.2014.PTu4B.5.

[10] U. Aygül, H. Egelhaaf, P. Nagel, M. Merz, S. Schuppler, Photodegradation of C PCPDTBT and Si-PCPDTBT: influence of the bridging atom on the stability of a low-band-gap polymer for solar cell application, ChemPhysChem 16 (2014) 428-435, https://doi.org/10.1002/cphc.201402494.

[11] M. Raja, N. Sedghi, S. Badriya, S.J. Higgins, G.C.R. Lloyd, W. Eccleston, Modelling of polymer Schottky diodes for real device applications, Proc. ESSDERC 2005 pp. 253-256, https://doi.org/10.1109/ESSDER.2005.1546633.

[12] N. Reitzel, D.R. Greve, K. Kjaer, P.B. Howes, M. Jayaraman, S. Savoy, R.D. McCullough, J.T. McDevitt, T. Bjørnholm, Self-assembly of conjugated polymers at the air/wate interface. Structure and properties of Langmuir and Langmuir-Blodgett films of amphiphilic regioregular polythiophenes, J. Am. Chem. Soc. 122 (2000) 5788-5800 https://doi.org/10.1021/ja9924501.

[13] D.A.P. Sarah R. Dennison, Frederick Harris, A Langmuir approach on monolayer interactions to investigate surface active peptides, Protein Pept. Lett. 17 (2010) 1363-1375, https://doi.org/10.2174/0929866511009011363.

[14] Gareth Roberts, Langmuir-Blodgett Films, 1st ed., 1990https://doi.org/10.1017 CBO9781107415324.004

[15] O.N.O. Jr, Langmuir-Blodgett films - properties and possible applications, Braz. J. Phys. 22 (1992) 60-69.

[16] M.C. Petty, C. Michael, Petty-Langmuir-Blodgett Films - An Introduction, 1996

[17] H. Borchert, Solar Cells Based on Colloidal Nanocrystals, 196th ed., 2014https://doi. org/10.1007/978-3-319-04388-3.

[18] N. Radychev, B. Kempken, C. Krause, J. Li, J. Kolny-Olesiak, H. Borchert, Photovoltaic response of hybrid solar cells with alloyed $\mathrm{ZnS}-\mathrm{CuInS}_{2}$ nanorods, Org. Electron. 21 (2015) 92-99.

[19] N.A. Rangel-Vázquez, Analysis and Molecular Characterization of Organic Materials for Application in Solar Cells, Structural Analysis using Computational Chemistry River Publishers, Aguascalientes 2016 September, pp. 79-104 , Chap 4

[20] J. Važgèla, M. Stephen, G. Juška, K. Genevičius, K. Arlauskas, Charge carrier transport properties in ternary Si-PCPDTBT: P3HT: PCBM solar cells, Lith. J. Phys. 57 (2017) 37-41.

[21] C. Soci, I.W. Hwang, D. Moses, Z. Zhu, D. Waller, R. Gaudiana, C.J. Brabec, A.J. Heeger, Photoconductivity of a low-bandgap conjugated polymer, Adv. Funct. Mater. 17 (2007) 632-636, https://doi.org/10.1002/adfm.200600199.

[22] M.L. Braunger, Fabrication and Electrical Characterization of Solar Cells From Organic Thin Films, FCT - UNESP, 2015

[23] H. Awada, Synthesis of Organic-Inorganic Hybrids for Photovoltaic Applications, Université de Pau et Pays de l'Adour, 2014.
[24] E.A. da Silva, V.J.R. de Oliveira, M.L. Braunger, C.J.L. Constantino, C. de A. Olivati, Poly (3 octylthiophene)/stearic acid Langmuir and Langmuir-Blodgett films: preparation and characterization, Mater. Res. 17 (2014) 1442-1448, https://doi.org/10.1590/ 1516-1439.288814.

[25] A. Nabok, Organic and Inorganic Nanostructures, 1st, ed., 2005.

[26] R.C. Sanfelice, V.C. Gonc, C. Av, T. Saocarlense, C.P. Cep, Langmuir and LangmuirSchaefer films of poly(3 hexylthiophene) with gold nanoparticles and gold nanoparticles capped with 1 octadecanethiol, J. Organomet. Chem. 118 (2014) 12944-12951.

[27] R. Singhal, A. Chaubey, T. Srikhirin, S. Aphiwantrakul, S.S. Pandey, B.D. Malhotra, Immobilization of glucose oxidase onto Langmuir-Blodgett films of poly 3 hexylthiophene, Curr. Appl. Phys. 3 (2003) 275-279, https://doi.org/10. 1016/S1567-1739(02)00215-8

[28] A. Modli, D. Bauman, The Langmuir-Blodgett technique as a tool for homeotropic alignment of fluorinated liquid crystals mixed with arachidic acid, Int. J. Mol. Sci. 12 (2011) 4923-4945, https://doi.org/10.3390/ijms12084923.

[29] J. George, L. Gaines, General, thermodynamic relationships for mixed insoluble monolayers, J. Colloid Interface Sci. 21 (1966) 315-319.

[30] H. Nakahara, C. Hirano, I. Fujita, O. Shibata, Interfacial properties in Langmuir monolayers and LB films of DPPC with partially fluorinated alcohol (F8H7OH), J. Oleo Sci. 62 (2013) 1017-1027.

[31] E.A da Silva, L Caseli, C. de A. Olivati, Organization of polythiophenes at ultrathin films mixed with stearic acid investigated with polarization-modulation infrared reflection-absorption spectroscopy, Colloids Surf. A Physicochem. Eng. Asp. 529 (2017) 628-633, https://doi.org/10.1016/j.colsurfa.2017.06.035.

[32] Z. Yu, J. Jin, Y. Cao, Characterization of the liquid-expanded to liquid-condensed phase transition of monolayers by means of compressibility, Langmuir 18 (2002) 4530-4531.

[33] S. Belegrinou, J. Dorn, M. Kreiter, K. Kita-tokarczyk, E. Sinner, W. Meier, Biomimetic supported membranes from amphiphilic block copolymers, Soft Matter 6 (2010) 179-186, https://doi.org/10.1039/b917318h.

[34] M.J.J.M. Conde, Characterization of the binary mixed monolayers of $\alpha$ tocopherol with phospholipids at the air-water interface, Biomed. Biochim. Acta 1828 (2013) 2410-2418, https://doi.org/10.1016/j.bbamem.2013.07.005.

[35] T. Haefele, K. Kita-Tokarczyk, W. Meier, Phase behavior of mixed Langmuir monolayers from amphiphilic block copolymers and an antimicrobial peptide, Langmuir 22 (2006) 1164-1172, https://doi.org/10.1021/la0524216.

[36] W. Callister Jr., Ciência e Engenharia de Materiais: Uma Introdução, 8th ed., 2012.

[37] G.L. Schulz, F.S.U. Fischer, D. Trefz, A. Melnyk, A. Hamidi-Sakr, M. Brinkmann, D. Andrienko, S. Ludwigs, The PCPDTBT family: correlations between chemical structure, polymorphism, and device performance, Macromolecules (2017)https://doi. org/10.1021/acs.macromol.6b01698.

[38] G. Chen, C. Chiang, D. Kekuda, S. Lan, C. Chu, K. Wei, Synthesis and characterization of a narrow-bandgap polymer containing alternating cyclopentadithiophene and diketo-pyrrolo-pyrrole units for solar cell applications, Polym. Chem. 48 (2010) 1669-1675, https://doi.org/10.1002/POLA

[39] H. Eckhardt, L.W. Shacklette, K.Y. Jen, R.L. Eisenbaumer, The electronic and electrochemical properties of poly (phenylene vinylenes) and poly(thienylene vinylenes): an experimental and theoretical study, J. Chem. Phys. 91 (1989) 1303-1315, https:// doi.org/10.1063/1.457153.

[40] P. Kumar, Oorganic Solar Cells. Device Physics, Processing, Degradation, and Prevention, 1st ed., 2016

[41] F.N. Crespilho, V. Zucolotto, O.N.O. Jr, F.C. Nart, Electrochemistry of layer-by-layer films: a review, Int. J. Electrochem. Sci. 1 (2006) 194-214.

[42] B.D. Mühlbacher, M. Scharber, M. Morana, Z. Zhu, D. Waller, R. Gaudiana, C. Brabec, High photovoltaic performance of a low-bandgap polymer, Adv. Mater. 18 (2006) 2884-2889, https://doi.org/10.1002/adma.200600160.

[43] E.W.T. Rhodes, E.B.A. Adibi, T. Asakura, T. Kamiya, F. Krausz, B. Monemar, H. Venghaus, H. Weber, H. Weinfurter, W.T. Rhodes, Springer Series in Springer Series in, 1 st ed., 2009.

[44] S. Kowalski, Direkt-Arylierung als neue Methode zur Synthese von Donor-AkzeptorCopolymeren, Universität Wuppertal, 2015http://nbn-resolving.de/urn/resolver.pl? urn=urn\%3Anbn\%3Ade\%3Ahbz\%3A468-20160113-113659-8.

[45] E.T. Samulski, J.M. Chem, www.rsc.org/materials]. Mater. Chem. 21 (2011) 16273-16652, https://doi.org/10.1039/c1jm12300a.

[46] K. Hashimoto, T. Koganezawa, K. Tajima, End-on orientation of semiconducting polymers in thin films induced by surface segregation of fluoroalkyl chains, J. Am. Chem. Soc 135 (2013) 9644-9647, https://doi.org/10.1021/ja4051179.

[47] T. Blythe, D. Bloor, Electrical Properties of Polymers, 2a edition, 2008. 